\title{
Acute Effects of Combination of IB and IC Antiarrhythmics for the Treatment of Ventricular Tachycardia
}

\author{
Barry E. Bleske, PharmD, Jeffrey Kluger, MD, Jane Fisher, RN, \\ and Moses S. S. Chow, PharmD, FCP
}

\begin{abstract}
There are limited data on the effects of Class IB and IC antiarrhythmic drug combination for the treatment of ventricular tachycardia. The present study evaluated this combination in 12 patients who had sustained ventricular tachycardia (SuVT) during programmed electrical stimulation (PES) and failed IC antiarrhythmic therapy. Following combination of lidocaine and a IC agent ( 7 with encainide and 5 with flecainide), two had no inducible ventricular tachycardia (VT) and one had nonsustained VT (NSVT). In seven of nine patients who still had SuVT, the mean VT cycle length increased $40 \pm 25$ msec post combination compared to IC antiarrhythmic therapy. Seven patients who had a favorable response to the initial combination ( $<10$ beats of NSVT, or $\geq 10$ beats of VT with $a>100$ msec increase in cycle length compared to baseline and no hemodynamic compromise) were then placed on IC + oral IB agent (5 with mexiletine, 2 with tocainide). Similar effects on VT inducibility and cycle length were observed following the oral combination. In conclusion, the addition of lidocaine to IC therapy produced favorable effects on induced ventricular tachycardia in $58 \%$ of patients compared to IC agent alone. Also, a positive PES response to lidocaine and IC therapy corresponded to a similar positive response when either mexiletine or tocainide was substituted for lidocaine.
\end{abstract}

$\mathbf{T}^{\mathrm{T}}$ he treatment of ventricular tachycardia is often difficult and may not be adequately controlled with a single antiarrhythmic agent. Reports in the literature suggest combination of antiarrhythmic agents, particularly class IA and IB agents (Vaughn Williams classification), are effective in treatment of ventricular arrhythmias. ${ }^{1.2 .3 .4}$ However, there is only limited data on the combination of IB (lidocaine) and IC (encainide, flecainide) antiarrhythmic agents. ${ }^{5}$ The purpose of this paper is to report our experience with this combination in 12 patients undergoing programmed electrical stimulation (PES) for the treatment of symptomatic ventricular tachycardia.

From the Departments of Medicine and Pharmacy, Hartford Hospital, Hartford, Connecticut and School of Medicine and Pharmacy, University of Connecticut, Farmington and Storrs Connecticut (Drs. Kluger, Fisher, and Chow), and the College of Pharmacy, University of Michigan, Ann Arbor, Michigan (Dr. Bleske). Address for Reprints: Jeffrey Kluger, MD, Division of Cardiology, Hartford Hospital, 80 Seymour Street, Hartford, CT 06115. Correspondence to: Barry E. Bleske, PharmD, Assistant Professor of Pharmacy, University of Michigan, College of Pharmacy, Ann Arbor, MI 48109-1065.

\section{METHODS}

Twelve patients with PES documented ventricular tachycardia uncontrolled with single antiarrhythmic agents were evaluated. The patient characteristics are described in Table I. Specifically, these patients were selected for lidocaine plus encainide or flecainide therapy based on previous PES response to these drugs. They either had an increase in cycle length of induced ventricular tachycardia following encainide or flecainide alone or no previous adverse outcome (decrease in ventricular tachycardia cycle length) following lidocaine during PES.

The PES protocol employed by our group has been described elsewhere. ${ }^{6}$ Briefly, the protocol involves ventricular pacing at three different cycle lengths $(600,500$, and 400 msecs) with introduction of single, double and triple extrastimulus until refractoriness. This is followed by rapid ventricular pacing for five captures up to a cycle length of $200 \mathrm{msec}$ or 2:1 capture or until sustained ventricular tachycardia is induced and reproducible. If ventricular tachycardia is not induced at the right ventricular apex, the 
TABLE I

\begin{tabular}{lc}
\hline \multicolumn{2}{c}{ Patient Characteristics } \\
\hline Patients & 12 \\
Males & 12 \\
Age (years) & $61 \pm 5$ \\
EF (\%) & $39 \pm 16$ \\
Cardiac Diagnosis: & 9 \\
Coronary Artery Disease & 2 \\
Hypertension & 1 \\
Cardiomyopathy & \\
Presenting Arrhythmias: & 5 \\
SuVT & 4 \\
Out of Hospital VF & 3 \\
NSVT & \\
\hline
\end{tabular}

SuVT = Sustained ventricular tachycardia; $N S V T=$ Nonsustained ventricular tachycardia; VF = Ventricular fibrillation;

stimulation protocol is repeated at the right ventricular outflow tract. Noninducible is defined as induction of less than ten extra beats. Nonsustained ventricular tachycardia is defined as induction of greater than nine extra beats but less than 30 seconds of ventricular tachycardia. Sustained ventricular tachycardia is defined as greater than $\mathbf{3 0}$ seconds of ventricular tachycardia or early termination by overdrive pacing or cardioversion because of hemodynamic compromise. Following the combination therapy, effective drug response is defined as less than ten beats of nonsustained ventricular tachycardia induced. A partial effective drug response is defined as induction of $\geq 10$ beats of nonsustained ventricular tachycardia or sustained ventricular tachycardia with significant lengthening of ventricular tachycardia cycle length (>100 msecs) from baseline and no hemodynamic compromise.

IC antiarrhythmic therapy consisted of either encainide up to $150 \mathrm{mg} /$ day or flecainide up to 500 $\mathrm{mg} /$ day or until adverse effects and/or excessive lengthening of the QRS interval $(>30 \%)$ were observed. Lidocaine was administered as a $3 \mathrm{mg} / \mathrm{kg}$ bolus over a 15 -minute period followed by a $2 \mathrm{mg}$ / minute continuous infusion.

Electrocardiogram (ECG) measurements were obtained by a trained research nurse who did not have knowledge of this study. Measurement of ECG intervals for all treatment groups were obtained from the same ECG leads. Refractory periods were measured following the first extrastimulus. Supine blood pressures were obtained with a blood pressure cuff and sphygmomanometer.

In addition to the above measurements, the mode of ventricular tachycardia induction and termina- tion were also assessed. The order of ascending difficulty in ventricular tachycardia induction were one extrastimulus, two extrastimulus, three extrastimulus, and rapid ventricular pacing. For termination, ventricular pacing was considered less difficult than external cardioversion to terminate ventricular arrhythmias. Proarrhythmic response was considered as the induction of an arrhythmia using two less extrastimuli $\mathrm{B}^{2}$ than previously required. In addition, the occurrence of sustained ventricular tachycardia when only nonsustained ventricular tachycardia was previously provoked and the induction of sustained ventricular tachycardia at a rate faster than previously induced were also considered as proarrhythmic responses.

\section{DATA ANALYSIS}

Data are presented as mean and standard deviation. Differences between treatment groups were tested by analysis of variance and Tukey's Studentized Range Test. A $P<.05$ was considered significant for all tests.

\section{RESULTS}

Prior to combination therapy, all 12 patients had induced sustained ventricular tachycardia at baseline (no drug). Eleven out of 12 patients also had induced sustained ventricular tachycardia while on either encainide $(\mathrm{N}=6)$ or flecainide $(\mathrm{N}=5)$ alone. One had nonsustained ventricular tachycardia with encainide therapy. Nine out of 12 patients had induced sustained ventricular tachycardia on lidocaine alone. The other three patients either clinically failed lidocaine therapy $(\mathrm{N}=2)$ or had a suspected adverse reaction to lidocaine and were not tested on lidocaine alone during PES.

In the nine patients who received lidocaine alone, there were no significant differences in cycle length compared to baseline ( $255 \pm 30$ vs $241 \pm 32 \mathrm{~ms}$ ). Also, there were no significant changes in ECG intervals or refractory periods compared to baseline. The mean serum concentration of lidocaine was 2.8 $\pm 0.7 \mathrm{mg} / \mathrm{L}$.

The effects of IC agent alone are presented in Tables II and III. Increases in sustained ventricular tachycardia cycle length, blood pressure, and PR and QRS intervals from baseline, but not refractory period, were observed. The mean dose of IC agents were $128 \pm 34 \mathrm{mg} /$ day for encainide and $280 \pm 75$ $\mathrm{mg} /$ day for flecainide. The serum concentration for flecainide was $0.52 \pm 0.31 \mathrm{mcg} / \mathrm{ml}$. No patients demonstrated a proarrhythmic response during IC therapy compared to baseline. 
TABLE ॥

\begin{tabular}{|c|c|c|}
\hline \multicolumn{3}{|c|}{$\begin{array}{l}\text { Cycle Length and Blood Pressure Response in } \\
\text { Patients With Sustained Ventricular Tachycardia } \\
\text { During IC plus Lidocaine Therapy }(n=9)\end{array}$} \\
\hline Treatment & $\begin{array}{l}\text { Cycle Length* } \\
\text { (ms) }\end{array}$ & $\begin{array}{c}\text { Systolic Pressure } \\
(\mathrm{mmHg})\end{array}$ \\
\hline $\begin{array}{l}\text { Baseline } \\
\text { Lidocaine }(n=7) \ddagger \\
\text { IC } \\
\text { IC + Lidocaine }\end{array}$ & $\begin{array}{l}257 \pm 45 \\
260 \pm 32 \\
366 \pm 66 \dagger \\
394 \pm 70 \dagger\end{array}$ & $\begin{array}{r}65 \pm 38 \\
54 \pm 37 \\
93 \pm 38 \\
107 \pm 22\end{array}$ \\
\hline \multicolumn{3}{|c|}{$\begin{array}{l}*=\text { Cycle length of induced sustained ventricular tachycardia. } \\
i=\text { P } P 0.05 \text { compared to baselile. } \\
\text { I Two patients were not tested on lidocaine alone, one patient clinically failed } \\
\text { lidocaine and one patient had a suspected adverse reaction. }\end{array}$} \\
\hline
\end{tabular}

With the addition of lidocaine to encainide, two patients became noninducible and one converted from sustained to nonsustained ventricular tachycardia (Table IV). No patients who received flecainide therapy converted from sustained ventricular tachycardia to nonsustained ventricular tachycardia or became noninducible. Nine patients still had sustained ventricular tachycardia but the mean cycle length in seven increased $40 \pm 25 \mathrm{msec}$, one had no change in cycle length (flecainide therapy) and one had a decrease in cycle length (encainide therapy) with stable blood pressure (Table II). Overall, seven out of 12 patients ( 4 encainide and 3 flecainide therapy) were judged to be responders based on abolition of sustained ventricular tachycardia or significant increase in ventricular tachycardia cycle length and stable blood pressure compared to baseline. The dose of encainide, flecainide, and lidocaine used during combination therapy remained the same or similar as previous single drug therapy.

For patients with sustained ventricular tachycardia the mode of induction with IC and lidocaine therapy was the same in five patients, harder in two,

\begin{tabular}{lc}
\hline \multicolumn{2}{c}{ TABLE IV } \\
\hline $\begin{array}{c}\text { Programmed Electrical Stimulation Response IC } \\
\text { plus Lidocaine Therapy }\end{array}$ \\
\hline Baseline & 12 Patients (SUVT) \\
IC & 11 Patients (SuVT) \\
& 1 Patient (NSVT) \\
IC + Lidocaine & 9 Patients (SUVT) \\
& 2 Patients (NI) \\
& 1 Patient (NSVT) \\
\hline
\end{tabular}

SuVT = Sustained ventricular tachycardia; $N I=$ Noninducible; $N S V T=$ Nonsustained ventricular tachycardia.

and easier in two compared to IC therapy alone. The mode of arrhythmia termination (either by cardioversion or rapid ventricular pacing) was the same in eight patients and harder in one when compared to IC agents alone. One patient required cardioversion secondary to accelerated ventricular tachycardia after attempted termination by rapid ventricular pacing. However, no patients were considered to have a proarrhythmic response.

Seven patients who were judged to be effective or partial responders to combination of either encainide or flecainide and lidocaine were continued on their respective IC agent along with an oral IB agent (mexiletine $n=5$, tocainide $n=2$ ). Following oral combination therapy similar PES responses were observed (Tables V and VI). The mode of induction and termination were also similar. The mean dose of encainide, flecainide, mexiletine and tocainide were $131 \pm 38 \mathrm{mg} /$ day, $316 \pm 160 \mathrm{mg} /$ day, $630 \pm 147$ $\mathrm{mg} /$ day, and $1500 \pm 300 \mathrm{mg} /$ day, respectively. The serum concentrations for flecainide, mexiletine and tocainide were $0.56 \pm 0.13 \mathrm{mcg} / \mathrm{ml}, 0.7 \pm 0.4 \mathrm{mcg} /$ $\mathrm{ml}$, and $5.2 \pm 0.7 \mathrm{mcg} / \mathrm{ml}$, respectively. Overall, five out of seven patients were discharged on combination therapy. The two patients who were not discharged on combination therapy required discon-

\begin{tabular}{|c|c|c|c|c|}
\hline \multicolumn{5}{|c|}{ TABLE III } \\
\hline \multicolumn{5}{|c|}{ Change in Refractory Period and ECG Intervals } \\
\hline & RP* & PR (ms) & QRS (ms) & QTe (ms) \\
\hline $\begin{array}{l}\text { Baseline } \\
\text { IC } \\
\text { IC + Lidocaine }\end{array}$ & $\begin{array}{l}246 \pm 23 \\
265 \pm 23 \\
265 \pm 25\end{array}$ & $\begin{array}{l}.19 \pm .04 \\
.23 \pm .04 t \\
.23 \pm .05 t\end{array}$ & $\begin{array}{l}.11 \pm .02 \\
.13 \pm .02 t \\
.14 \pm .02 \dagger\end{array}$ & $\begin{array}{l}.43 \pm .04 \\
.47 \pm .06 \\
.45 \pm .04\end{array}$ \\
\hline - = Refractory period of fir & & $t=1$ & red to baseline. & \\
\hline
\end{tabular}




\section{TABLE V}

\begin{tabular}{|c|c|c|}
\hline \multicolumn{3}{|c|}{$\begin{array}{l}\text { Sustained Ventricular Tachycardia Cycle Length and } \\
\text { Blood Pressure Response to IB, IC, IC + IB, and IC } \\
\text { + oral IB Antiarrhythmic Agents }(n=4)\end{array}$} \\
\hline Treatment & Cycle Length* (ms) & $\begin{array}{l}\text { Systolic Pressure } \\
(\mathbf{m m H g})\end{array}$ \\
\hline $\begin{array}{l}\text { Baseline } \\
\text { Lidocaine } \\
\text { IC } \\
\text { IC + Lidocaine } \\
\text { IC + Oral IB }\end{array}$ & $\begin{array}{l}247 \pm 22 \\
259 \pm 8 \\
348 \pm 37 \dagger \\
388 \pm 33 \dagger \\
410 \pm 40 \dagger\end{array}$ & $\begin{array}{r}52 \pm 44 \\
73 \pm 40 \\
114 \pm 37 \\
121 \pm 34 \\
122 \pm 17\end{array}$ \\
\hline
\end{tabular}

tinuation of their therapy secondary to adverse effects (rash, and CNS disturbances).

\section{DISCUSSION}

Our present study indicates that the addition of lidocaine to either encainide or flecainide can enhance antiarrhythmic response. Overall, seven out of 12 patients were judged to have a favorable response, i.e., effective or partially effective to the combination therapy. This high success rate can be attributed to the definition of favorable response, which include those who had a significant increase in ventricular tachycardia cycle length with no symptoms. Waller et al., showed that patients who had an increase in ventricular tachycardia cycle length of greater than $100 \mathrm{msec}$ and no severe symptoms had a long-term outcome similar to patients who were noninducible. ${ }^{7}$ If a stricter criteria, noninducibility, was used to define favorable response, the response rate from our combination therapy would be much lower $(2 / 12$ patients responding). This low response rate is similar to a previous report by Ross et al., who showed that oral encainide plus lidocaine produced no increase in efficacy compared to baseline when noninducibility was used as an endpoint. ${ }^{5}$

Our experience also indicates that a favorable response to combination of lidocaine and IC agents predicts a similar response to combination of oral IB and IC agents. This supports a previous study by Podrid and Lown who showed that lidocaine is predictive of tocainide's response in $78 \%$ of patients. ${ }^{8}$ It should be pointed out that in our study, only positive responses were evaluated. No information is available about negative concordance between lidocaine and oral IB drugs in our patient population.
Although proarrhythmic response to IC therapy has been reported to occur none were seen during our study. ${ }^{9-12}$ This is due to our patient selection which excluded patients that did not have a favorable response to IC therapy. Further, no proarrhythmic responses were seen with the addition of lidocaine to IC therapy. This was also demonstrated in previous studies. ${ }^{13,14}$ Other studies have shown that lidocaine may prevent and/or treat proarrhythmic responses to flecainide. ${ }^{14,15}$ Thus, the addition of lidocaine to IC therapy appears to be safe and may prevent proarrhythmic responses in addition to enhancing therapeutic effects. ${ }^{13.14}$ However, further data is needed to substantiate this.

A potential concern with the results of our study is the general grouping of IB and IC antiarrhythmic agents, with the assumption that all IB or IC agents produce similar results. Previous studies showed that neither mexiletine nor tocainide predicts response to each other. ${ }^{16}$ However, within the IC group, we observed similar response to encainide and flecainide with or without lidocaine combination. The ventricular tachycardia cycle length post encainide and flecainide increased $127 \pm 37 \mathrm{msec}$ and $123 \pm 90 \mathrm{msec}$ from baseline, respectively. With the addition of lidocaine, the ventricular tachycardia cycle length post lidocaine-encainide and lidocaine-flecainide increased $151 \pm 79 \mathrm{msec}$ and 141 $\pm 83 \mathrm{msec}$ from baseline respectively. Unfortunately, due to the low number of patients, no comparisons can be made on oral IB and IC combination therapy.

In summary, our small series of patients showed that the addition of lidocaine to either flecainide or encainide improved therapeutic response when

TABLE VI

Programmed Electrical Stimulation Response: IC vs IC + Lidocaine vs IC + Oral IB Therapy

\begin{tabular}{ll}
\hline Baseline & 7 Patients (SuVT) \\
IC & 6 Patients (SuVT) \\
& 1 Patient (NSVT) \\
IC + Lidocaine & 4 Patients (SuVT) \\
& 2 Patients (NI) \\
1 Patient (NSVT) \\
IC + Oral IB & 4 Patients (SuVT) \\
& 2 Patients (NI) \\
& 1 Patient (NSVT) \\
\hline
\end{tabular}

SUVT = Sustained ventricular tachycardia; $N I=$ Noninducible; $N S V T=$ Non . sustained ventricular tachycardia. 
modification of sustained ventricular tachycardia is used as an endpoint. Furthermore, a favorable response observed with a combination of lidocaine and flecainide or encainide predicted a similar response to a combination of oral IB (mexiletine or tocainide) and IC (flecainide or encainide) agents. Therefore, IB and IC combination therapy may be a viable alternative for patients refractory to single antiarrhythmic agents. Further studies, however, are needed to verify our favorable results for IB and IC combination therapy.

\section{REFERENCES}

1. Greenspan AM, Spielman SR, Webb CR, Sokoloff NM, Pae AP, Horowitz LN: Efficacy of combination therapy with mexiletine and a type IA agent for inducible ventricular tachyarrhythmias secondary to coronary artery disease. Am J Cardiol 1985;56:277284

2. Duff HJ, Roden D, Primm RK, Oates JA, Woosley RL: Mexiletine in the treatment of resistant ventricular arrhythmias: en hancement of efficacy and reduction of dose-related side effects by combination with quinidine. Circulation 1983;67(5):11241128.

3. Kim SG, Mercando AD, Fisher JD: Combination of tocainide and quinidine for better tolerance and additive effects in patients with coronary artery disease. J Am Coll Cardiol 1987;9:1369-1374.

4. Vaughn Williams EM: A classification of antiarrhythmic actions reassessed after a decade of new drugs. J Clin Pharmacol 1984;24:129-147.

5. Ross DL, Sze DY, Keefe DL, Swerdlow CD, Echt DS, Griffen JC, Winkle RA, Mason JW: Antiarrhythmic drug combinations in the treatment of ventricular tachycardia efficacy and electrophysiological effects. Circulation 1982;66(6):1205-1210.

6. Capparelli EV, Kluger J, Regnier JC, Chow MSS: Clinical and electrophysiological effects of flecainide in patients with refractory ventricular tachycardia. J Clin Pharmacol 1988;28:268-275.

7. Waller TJ, Kay HR, Spielman SR, Kutalek SP, Greenspan AM, Horowitz LN: Reduction in sudden death and total mortality by antiarrhythmic therapy evaluated by electrophysiologic drug testing: Criteria of efficacy in patients with sustained ventricular tachyarrhythmias. J Am Coll Cardiol 1987;10:83-89.

8. Podrid PJ, Lown B: Tocainide for refractory symptomatic ventricular arrhythmias. Am J Cardiol 1982;49:1279-1286.

9. Somberg JC, Tepper D: Flecainide: a new antiarrhythmic agent. Am Heart J 1986;112:808-813.

10. Morganroth J, Anderson JL, Gentzkow GD: Classification by type of ventricular arrhythmia predicts frequency of adverse cardiac events from flecainide. J Am Coll Cardiol 1986;8:607-615.

11. Somberg JC, Zanger D, Levine E, Tepper D: Encainide: a new and potent antiarrhythmic. Am Heart J 1987;114:826-835.

12. Winkle RA, Mason JW, Griffin JC, Ross D: Malignant ventricular tachyarrhythmias associated with the use of encainide. Am Heart J 1981;102:857-864.

13. Landau S, Hogan C, Somberg J: Evaluation of a possible electrophysiologic interaction of flecainide and lidocaine (abstr). $J$ Clin Pharmacol 1988;28:910.

14. Somberg JC, Capasso J, Leon L, Tepper D: Does lidocaine effect flecainide induced ventricular arrhythmias (abstr). Clin Res 1987;35:328A.

15. Maza SR, Wynn J, Schwartz J, Zanger D, Keefe D, Miura D, Somberg JC: Treatment of flecainide induced ventricular tachycardia with lidocaine (abstr). J Am Coll Cardiol 1986;7:159A.

16. Hession M, Blum R, Podrid PJ, Lampert S, Stein J, Lown B: Mexiletine and tocainide: Does response to one predict response to the other? J Am Coll Cardiol 1986;7:338-343. 